\title{
Boundary shape identification in two-dimensional electrostatic problems using SQUIDs
}

\author{
H.T. BANKS ${ }^{1}$ and F. KOJIMA ${ }^{2}$
}

\begin{abstract}
This paper is concerned with a quantitative nondestructive evaluation of conductors using superconducting quantum interference devices ( SQUIDs ). A measurement system is described for an electrical potential problem with an unknown boundary. Domain identification is discussed within the theoretical framework of a parameter estimation problem for the electrostatic field analysis. Applying the method of mappings to the problem considered here, we present computational methods, including theoretical convergence results for the associated finite dimensional problem identification techniques.
\end{abstract}

\section{INTRODUCTION}

Recently, demand has grown for assessing the structural integrity of materials used in nuclear power plants using advanced sensor technologies. An important effort on such problems entails quantitative nondestructive evaluation methods in magnetic flux imaging techniques. These methods involve an attempt to characterize structural flaws or defects which may not be detectable by visual inspection. SQUIDs ( superconducting quantum interference devices ) have the potential to detect material defects in conductors due to their extremely high magnetic flux sensitivity [1]. In this paper, we propose a computational method for recovering defect shapes with magnetic flux density data from high critical temperature SQUIDs (HTc-SQUIDs).

In the proposed nondestructive test, a stationary current density is applied to the conductor inspected. The magnetic flux density can be measured from a SQUID sensor located near the conductor. Figure 1 illustrates the inspection process using an HTc-SQUID. As shown in Fig. 1, the existence of a defect ( represented by a nonconducting volume ) corrupts the current flows inside the conductor and, as a result, this material defect can be

\footnotetext{
${ }^{1}$ Center for Research in Scientific Computation, North Carolina State University, Raleigh, NC 276958205, USA. E-mail:htbanks@eos.ncsu.edu

${ }^{2}$ Department of Mechanical Engineering, Osaka Institute of Technology, 5-16-1, Ohmiya, Asahi-ku, Osaka 535-8585 JAPAN. E-mail:kojima@med.oit.ac.jp
} 
detected as the perturbation of magnetic flux data. It is well-known that a mathematical model for this nondestructive test can be derived from Maxwell's equations. By Faraday's law and Gauss's law, the electrical potential E satisfies

$$
\begin{aligned}
& \nabla \times \mathbf{E}=0 \\
& \nabla \cdot \epsilon \mathbf{E}=\rho
\end{aligned}
$$

where $\epsilon$ and $\rho$ denote the permittivity and the charge density of the sample specimen, respectively. We introduce the electrical scalar potential $\phi$ such that

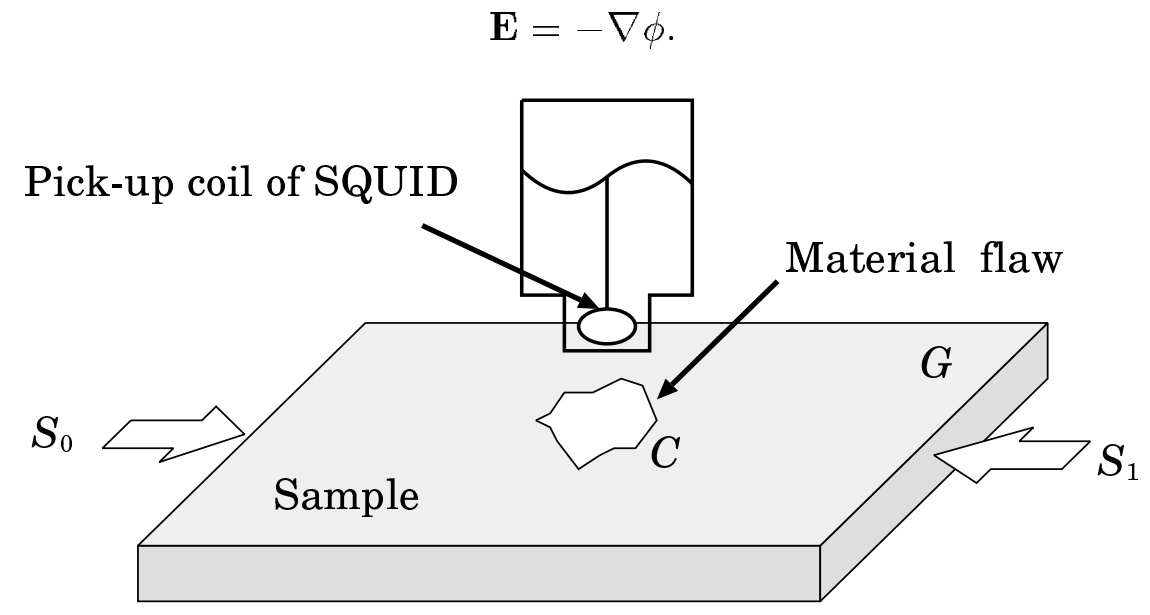

Fig. 1 Inspection process using HTc-SQUID

Assuming that there is no charge density inside the material and that the conductor is a homogeneous plate, we can rewrite Eqs. (1)-(2) as the Laplace equation

$$
\nabla^{2} \phi=0 \quad \text { in } \quad G_{3} \subset R^{3},
$$

with non-homogeneous boundary conditions

$$
\phi=v \quad \text { on } \quad \partial G_{3}^{D}, \quad \nabla \phi \cdot \hat{n}=0 \quad \text { on } \quad \partial G_{3}^{N} \cup \partial G_{3}^{C},
$$

where $G_{3}$ and $\partial G_{3}=\partial G_{3}^{D} \cup \partial G_{3}^{N} \cup \partial G_{3}^{C}$ denote, respectively, the domain and the boundary of the conductor with a flaw. More specifically, the boundary $\partial G_{3}^{D} \cup \partial G_{3}^{N}$ denotes the exterior boundary of the conductor and $\partial G_{3}^{C}$ characterizes the defect shape. In the above equations, $v$ denotes the prescribed electrical potentials on $\partial G_{3}^{D}$. The measurement of magnetic flux density by HTc-SQUIDs is described by Biot-Savart's law [2], for $x=$ $\left(x_{1}, x_{2}, x_{3}\right) \in R^{3}-\overline{G_{3}}$,

$$
B(x)=-\frac{\sigma_{0} \mu_{0}}{4 \pi} \int_{G_{3}} \frac{\nabla \phi \times\left(x-x^{\prime}\right)}{\left|x-x^{\prime}\right|^{3}} d x^{\prime}
$$


where $\sigma_{0}$ and $\mu_{0}$ denote the electrical conductivity and magnetic permeability of the conductor, respectively. Such measurements can be obtained by detecting voltage changes in SQUID magnetmetors. We assume that the measurements are only made on the vertical direction $\left(x_{3}\right.$-axis). Since the voltage change can be measured through the pickup coil, we can only take the average of the magnetic flux density at the sensor location. Suppose that $x_{p} \in R^{3}-\overline{G_{3}}$ is the location of sensor and $S_{p}$ denotes the region of the pickup coil with the center $x_{p}$. The problem considered here is that of identifying, from input and output data $\left\{v, B\left(x_{p}\right)\right\}$, the geometrical shape of the defect. If we assume that the conductor is a thin plate and that the defect to be identified is uniform in the $x_{3}$ direction, the corresponding electrical potential becomes

$$
\nabla \phi \approx\left(\frac{\partial \phi}{\partial x_{1}}, \frac{\partial \phi}{\partial x_{2}}, 0\right)
$$

Then the observation at $x_{p}$ can be approximated by

$$
B_{x_{3}}\left(x_{p}\right) \approx Y_{x_{p}}=-\frac{\sigma_{0} \mu_{0}}{4 \pi\left|S_{p}\right|} \int_{S_{p}}\left[\int_{G_{3}}\left\{\left(x_{2}-x_{2}^{\prime}\right) \frac{\partial \phi}{\partial x_{1}^{\prime}}-\left(x_{1}-x_{1}^{\prime}\right) \frac{\partial \phi}{\partial x_{2}^{\prime}}\right\}\left|x-x^{\prime}\right|^{-3} d x^{\prime}\right] d x,
$$

where $\left|S_{p}\right|$ denotes the crosssectional area of the HTc-SQUID. From our assumptions, the identification problem is well approximated by using a 2-D spatial domain. Thus, in this paper, our attention is restricted to a domain identification problem in two dimensions.

This paper is organized as follows. In Section 2, the mathematical model for the system is described by the Laplace equation in a two-dimensional spatial domain. The measurements are derived from Biot-Savart's law. Then these problems are treated as domain identification problems in electrostatic field analysis. In Section 2, we also formulate this problem in an abstract setting in a Hilbert space. The ideas proposed in Banks et. al., [3][4] using the "method of mappings" are adopted to the problem considered here. In Section 3, for computational purposes, we approximate the Hilbert space by finite dimensional subspaces and we discuss convergence analysis for the approximate identification problems. A practical computational algorithm is proposed briefly in the last section.

\section{PROBLEM FORMULATION AND BASIC ASSUMPTIONS}

Let $G$ be the sectional plane of the sample specimen $G_{3}$ as shown in Fig. 1 . Let $q$ be a constant vector which characterizes an unknown defect domain where $q$ belongs to an admissible parameter set $Q$ such that

(H-0) The admissible parameter set $Q$ is a compact subset of $R^{M}$.

We consider a bounded domain $C_{q}\left(\subset G \subset R^{2}\right)$ which is parametrized by unknown values of $q$. As depicted in Fig. 2, the bounded domain $G_{q}$ is considered as the system domain 
such that $G_{q}=G-\overline{C_{q}}$. Corresponding to the boundary $\partial G_{3}^{D}, \partial G_{3}^{N}$ and $\partial G_{3}^{C}$ in three dimensions, we assume the boundary of $G_{q}$ consists of the following components:

$$
\partial G_{q}=\cup_{i=1}^{4} \partial G_{i} \bigcup \partial C_{q}
$$

We further assume that $\operatorname{meas}\left(G_{q}\right) \geq \delta>0$, uniformly for $q \in Q$, for some positive $\delta$.

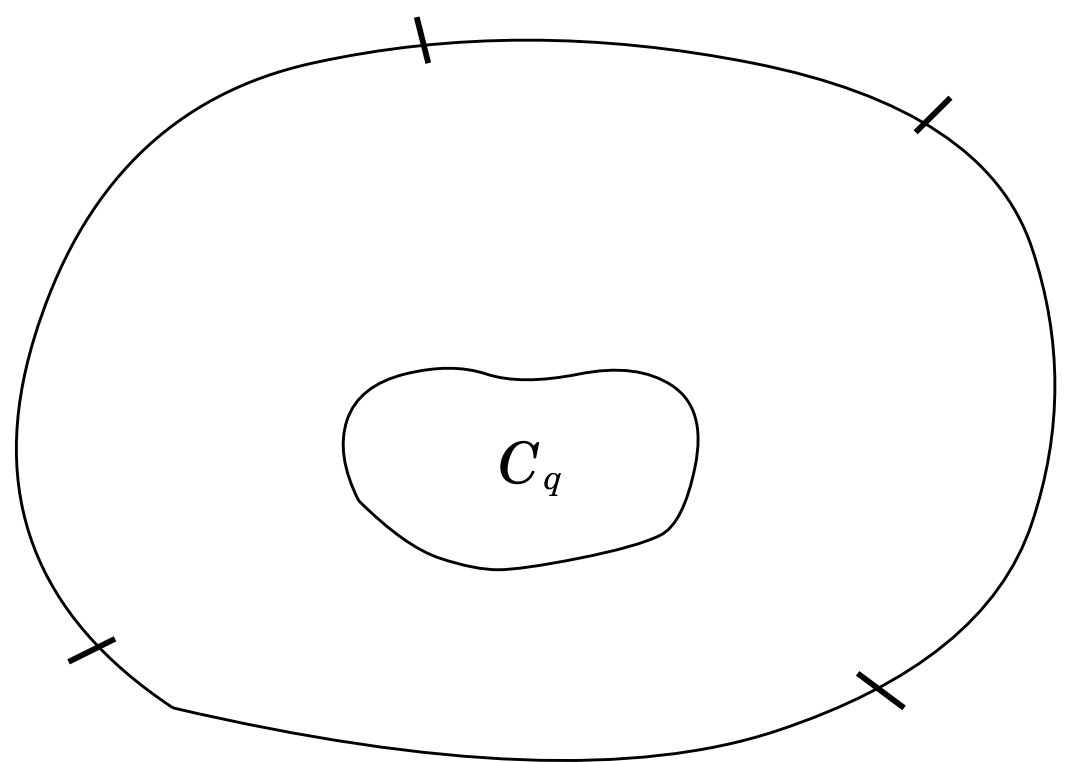

Fig. 2 Spatial domain $G$ in two dimensions

Thus, from Eqs.(3) and (4), the system can be rewritten by

$$
\begin{aligned}
\nabla^{2} \phi & =0 \quad \text { in } \quad G_{q} \\
\phi & =v_{i} \quad \text { on } \quad \partial G_{i}, \quad i=1,3, \\
\nabla \phi \cdot \hat{n} & =0 \quad \text { on } \quad \partial G_{i} \cup \partial C_{q}, \quad i=2,4 .
\end{aligned}
$$

Suppose that the SQUID sensor is scanned on the $x_{1}-x_{2}$ plane and measurements are made at $\left\{x_{p}^{i}=\left(x_{1 p}^{i}, x_{2 p}^{i}\right)\right\}_{i=1}^{m}$. Let $S_{p}^{i}$ be the region of the pick-up coil whose center is located at $x_{p}^{i}$. We also assume that the distance between the surface of the plate and SQUID sensor is taken as $h$. Then, from (5), the observation in case of two-dimensions is described by

$$
\begin{aligned}
& Y_{p}^{i}=-\frac{\sigma_{0} \mu_{0} \Delta}{4 \pi\left|S_{p}^{i}\right|} \int_{S_{p}^{i}}\left[\int_{G_{q}}\left\{\left(x_{2}-x_{2}^{\prime}\right) \frac{\partial \phi}{\partial x_{1}^{\prime}}-\left(x_{1}-x_{1}^{\prime}\right) \frac{\partial \phi}{\partial x_{2}^{\prime}}\right\}\right. \\
&\left.\times\left\{\left(x_{1}-x_{1}^{\prime}\right)^{2}+\left(x_{2}-x_{2}^{\prime}\right)^{2}+h^{2}\right\}^{-3 / 2} d x_{1}^{\prime} d x_{2}^{\prime}\right] d x_{1} d x_{2}, \\
& \text { for } \quad i=1,2, \cdots, m
\end{aligned}
$$


where $\Delta$ denotes the thickness of the plate. Let $\phi^{*}$ be an arbitrary smooth function on $G_{q}$ satisfying

$$
\phi^{*}=v_{i} \quad \text { in } \quad \partial G_{i}, \quad i=1,3 .
$$

Then the system (6)-(8) is equivalent to the following boundary value problem :

$$
\phi=u+\phi^{*}
$$

where $u$ is the solution of

$$
\begin{aligned}
\nabla^{2} u & =-\nabla^{2} \phi^{*} \quad \text { in } \quad G_{q} \\
u & =0 \text { on } \partial G_{i}, \quad i=1,3, \\
\nabla u \cdot \hat{n} & =-\nabla \phi^{*} \cdot \hat{n} \quad \text { on } \quad \partial G_{i} \cup \partial C_{q}, \quad i=2,4 .
\end{aligned}
$$

Let $V_{q}$ be the closed subset of functions in $H^{1}\left(G_{q}\right)$ satisfying the homogeneous boundary conditions on $\partial G_{i}, i=1,3$, i.e.,

$$
V_{q}=\left\{\psi \mid \psi \in H^{1}\left(G_{q}\right), \psi=0 \text { on } \partial G_{i}, i=1,3\right\} .
$$

endowed with the norm

$$
|\psi|_{V_{q}} \stackrel{\text { def }}{=} \int_{G_{q}}|\nabla \psi|^{2} d x
$$

Lemma 1. Suppose that

(C-1) $\phi^{*}$ is an element of $V_{q}^{v}$, the inhomogeneous analogue of $V_{q}$ defined by

$$
V_{q}^{v}=\left\{\psi \mid \psi \in H^{1}\left(G_{q}\right), \psi=v_{i} \text { on } \partial G_{i}, i=1,3\right\}
$$

Then, for every $q \in Q$, there exists a unique solution $\phi(q) \in V_{q}^{v}$ of $(6)$-(8) in the sense that

$$
u=\phi-\phi^{*} \in V_{q}
$$

satisfies (10)-(12). The solution $u$ of (10)-(12) is in the weak sense, i. e.,

$$
\sigma(q)(u, \psi)=-\sigma(q)\left(\phi^{*}, \psi\right) \text { for } \psi \in V_{q}
$$

Here $\sigma(q)(\cdot, \cdot)$ denotes a sesquilinear form on $V_{q} \times V_{q}$ defined by

$$
\sigma(q)(\varphi, \psi) \stackrel{\text { def }}{=} \int_{G_{q}} \nabla \varphi \cdot \nabla \psi d x .
$$

Moreover, we have

$$
|u(q)|_{V_{q}} \leq K_{1}\left|\phi^{*}\right|_{V_{q}}
$$

where $K_{1}$ is a constant independent of $q$. 
Remark. We note that even though $\phi^{*}$ is in $V_{q}^{v}$ and not in $V_{q}$, the value of $\sigma(q)\left(\phi^{*}, \psi\right)$ for $\psi \in V_{q}$ can be defined as in (15) and the value of $\left|\phi^{*}\right|_{V_{q}}$ is well defined as in (13). We tacitly assume in the statement of Lemma 1 and in subsequent discussions that such an interpretation is understood.

Proof. From (15), for arbitrary $\varphi \in V_{q}, \sigma(q)$ is V-elliptic with constant $C_{1}=1$ which is independent of $q$ since

$$
\sigma(q)(\varphi, \varphi)=\int_{G_{q}}|\nabla \varphi|^{2} d x=|\nabla \varphi|_{L_{2}\left(G_{q}\right)}^{2}=|\varphi|_{V_{q}}^{2}
$$

Since we have

$$
|\sigma(q)(\varphi, \psi)| \leq|\varphi|_{V_{q}}|\psi|_{V_{q}}
$$

the linear functional $-\sigma(q)\left(\phi^{*}, \cdot\right)$ is bounded on $V_{q}$, i. e.,

$$
\left|-\sigma(q)\left(\phi^{*}, \varphi\right)\right| \leq|\varphi|_{V_{q}}\left|\phi^{*}\right|_{V_{q}} \quad \text { for } \quad \varphi \in V_{q}
$$

From (16) and (17), we can apply the Lax-Milgram lemma, taking the Hilbert space $V_{q}$, sesquilinear form $\sigma(q)(\cdot, \cdot)$, and linear functional $-\sigma(q)\left(\phi^{*}, \cdot\right)$, respectively. Hence, for each $q \in Q$, there exists a unique solution $u \in V_{q}$ in the sense of (14). Similarly, from (14), (16) and (17), we have

$$
|u|_{V_{q}}^{2} \leq\left|-\sigma(q)\left(\phi^{*}, u\right)\right| \leq\left|\phi^{*}\right|_{V_{q}}|u|_{V_{q}}
$$

from which follows

$$
|u|_{V_{q}} \leq\left|\phi^{*}\right|_{V_{q}}
$$

thus completing the proof.

For convenience of theoretical developments, we use the polar coordinate system $x=(r, \theta)$ instead of the Cartesian coordinate $x=\left(x_{1}, x_{2}\right)$ in the sequel. To this end, we introduce the unknown defect function $c(\theta, q)$ and the prescribed outer boundary function $l(\theta)$. These functions are assumed to be defined on $(0,2 \pi)$ and are $2 \pi$-periodic, respectively. As depicted in Fig. 3, it is further assumed that the system domain $G_{q}$ can be described by

$$
G_{q}=\{(r, \theta) \mid 0<\theta<2 \pi, c(\theta, q)<r<l(\theta)\},
$$

and hence $\theta \rightarrow c(\theta, q)$ is a parameterized function which is assumed to characterize the unknown defect shape. The boundary of $G_{q}$ is also defined by

$$
\begin{aligned}
& \partial G_{i}=\left\{(r, \theta) \mid \bar{\theta}_{i-1}<\theta<\bar{\theta}_{i}, r=l(\theta)\right\} \text { for } i=1,2,3,4, \\
& \partial C_{q}=\{(r, \theta) \mid 0<\theta<2 \pi, r=c(\theta, q)\},
\end{aligned}
$$

where $\bar{\theta}_{0}=0$ and $\bar{\theta}_{4}=2 \pi$, respectively. The sesquilinear form (15) can be rewritten as

$$
\sigma(q)(\varphi, \psi)=\int_{0}^{2 \pi} \int_{c(\theta, q)}^{l(\theta)}\left(r \frac{\partial \varphi}{\partial r} \frac{\partial \psi}{\partial r}+\frac{1}{r} \frac{\partial \varphi}{\partial \theta} \frac{\partial \psi}{\partial \theta}\right) d r d \theta
$$




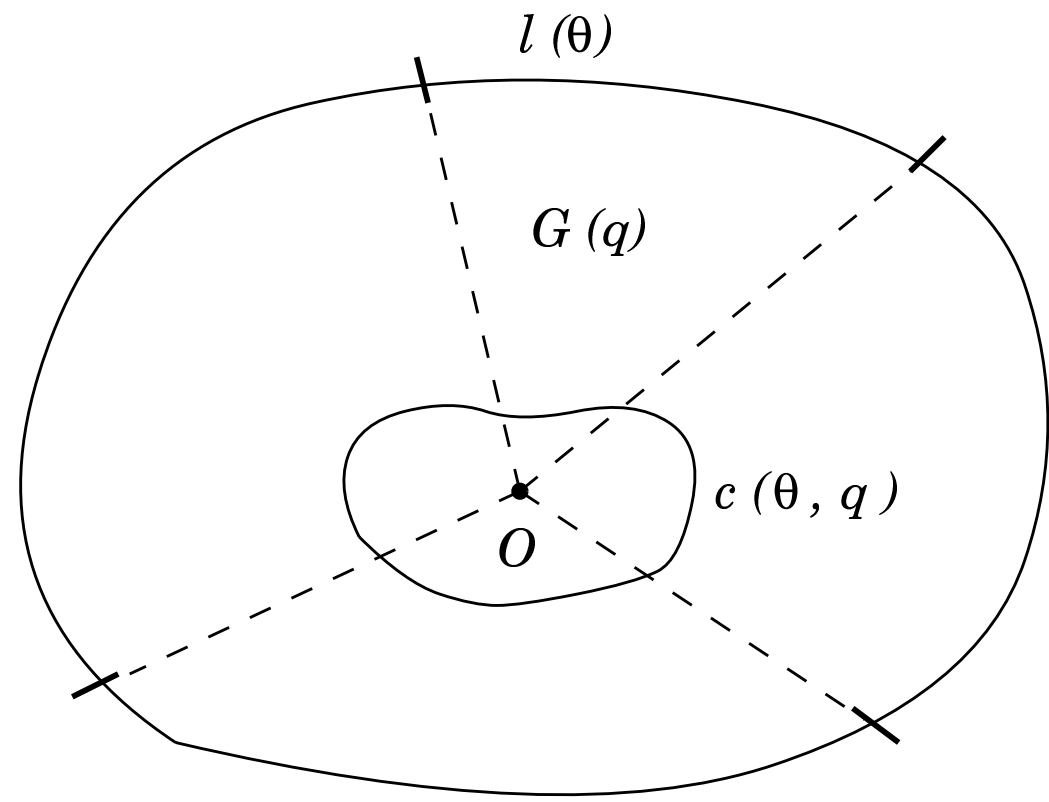

Fig. 3 The spatial domain $G$ and its boundary using the polar coordinate system

For the discussions here, we restrict the geometrical structure of the boundary $\partial G_{q}$ by imposing the following hypotheses:

(H-1) For each $q \in Q, c(\cdot, q) \in W_{\infty}^{1}(0,2 \pi)$ and $l \in W_{\infty}^{1}(0,2 \pi)$.

(H-2) There are constants $\beta_{1}$ and $\beta_{2}$ such that, for $q \in Q$,

$$
0<\beta_{1} \leq c(\theta, q) \leq \beta_{2}<l(\theta)<\infty \text { for } \theta \text { in }(0,2 \pi)
$$

(H-3) There exists a function $d: Q \times Q \rightarrow R^{1}$ with $d(q, \tilde{q}) \rightarrow 0$ as $|q-\tilde{q}| \rightarrow 0$ such that

$$
|c(\cdot, q)-c(\cdot, \tilde{q})|_{1, \infty} \leq d(q, \tilde{q}) \quad \text { for } q, \tilde{q} \in Q,
$$

where $|\cdot|_{1, \infty}$ denotes the norm of $W_{\infty}^{1}$.

Following standard procedures in the method of mapping techniques ([3],[4],[9]), we introduce the affine mapping $x=T(q) \tilde{x}$ where $x=(r, \theta)$ and $\tilde{x}=(\tilde{r}, \tilde{\theta})$. The precise form of $T(q)$ in this paper is given by

$$
\left\{\begin{array}{l}
r=\gamma(q ; \tilde{r}, \tilde{\theta})=\{c(\tilde{\theta}, q)-l(\tilde{\theta})\}\{\bar{c}-l(\tilde{\theta})\}^{-1}\{\tilde{r}-l(\tilde{\theta})\}+l(\tilde{\theta}) \\
\theta=\tilde{\theta}
\end{array} .\right.
$$

Let $\tilde{G}$ be the reference domain given by

$$
\tilde{G}=\{\tilde{x}=(\tilde{r}, \tilde{\theta}) \mid 0<\tilde{\theta}<2 \pi, \bar{c}<\tilde{r}<l(\tilde{\theta})\}
$$

which is independent of the parameter $q$ as depicted in Fig. 4. 


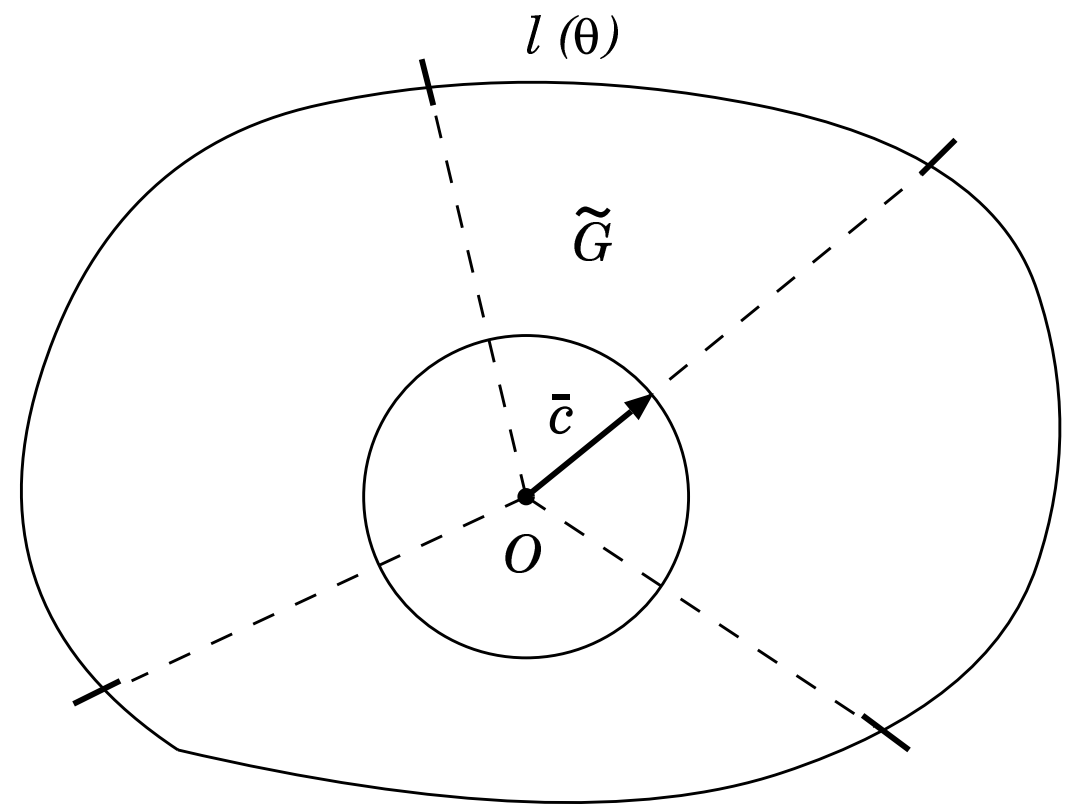

Fig. 4 The reference domain $\tilde{G}$

Then, under this coordinate change $T(q)$, the reference domain $\tilde{G}$ is transformed into the unknown domain $G_{q}$. Let us define the Hilbert space defined on $\tilde{G}$,

$$
\tilde{V}=\left\{\psi \mid \psi \in H^{1}(\tilde{G}), \psi=0 \text { on } \partial G_{i}, i=1,3\right\}
$$

endowed with the norm

$$
|\psi|_{\tilde{V}} \stackrel{\text { def }}{=} \int_{0}^{2 \pi} \int_{\bar{c}}^{l(\theta)}\left\{\left|\frac{\partial \psi}{\partial \tilde{r}}\right|^{2}+\left|\frac{\partial \psi}{\partial \tilde{\theta}}\right|^{2}\right\} d \tilde{r} d \tilde{\theta} .
$$

Noting that

$$
\begin{aligned}
\nabla T(q) & =\left[\begin{array}{cc}
\partial \gamma / \partial \tilde{r} & \partial \gamma / \partial \tilde{\theta} \\
0 & 1
\end{array}\right] \\
|\nabla T(q)| & =\frac{\partial \gamma}{\partial \tilde{r}}
\end{aligned}
$$

we have

$$
\begin{aligned}
\frac{\partial \varphi}{\partial r} & =\left(\frac{\partial \gamma}{\partial \tilde{r}}\right)^{-1} \frac{\partial \tilde{\varphi}}{\partial \tilde{r}} \\
\frac{\partial \varphi}{\partial \theta} & =-\left(\frac{\partial \gamma}{\partial \tilde{r}}\right)^{-1} \frac{\partial \gamma}{\partial \tilde{\theta}} \frac{\partial \tilde{\varphi}}{\partial \tilde{r}}+\frac{\partial \tilde{\varphi}}{\partial \tilde{\theta}} \\
d r d \theta & =\frac{\partial \gamma}{\partial \tilde{r}} d \tilde{r} d \tilde{\theta}
\end{aligned}
$$


Hence (18) can be transformed into the sesquilinear form on $\tilde{V} \times \tilde{V}$ as follows:

$$
\begin{aligned}
\tilde{\sigma}(q)(\varphi, \psi) \stackrel{\text { def }}{=} & \int_{0}^{2 \pi} \int_{\bar{c}}^{l(\theta)}\left\{a_{1}(q) \frac{\partial \varphi}{\partial \tilde{r}} \frac{\partial \psi}{\partial \tilde{r}}+a_{2}(q)\left(\frac{\partial \varphi}{\partial \tilde{r}} \frac{\partial \psi}{\partial \tilde{\theta}}+\frac{\partial \varphi}{\partial \tilde{\theta}} \frac{\partial \psi}{\partial \tilde{r}}\right)\right. \\
& \left.+a_{3}(q) \frac{\partial \varphi}{\partial \tilde{\theta}} \frac{\partial \psi}{\partial \tilde{\theta}}\right\} d \tilde{r} d \tilde{\theta}
\end{aligned}
$$

where

$$
\begin{aligned}
& a_{1}(q) \stackrel{\text { def }}{=} \gamma(q)^{-1}\left\{\frac{\partial \gamma}{\partial \tilde{r}}(q)\right\}^{-1}\left[\gamma^{2}+\left\{\frac{\partial \gamma}{\partial \tilde{\theta}}(q)\right\}^{2}\right], \\
& a_{2}(q) \stackrel{\text { def }}{=}-\gamma(q)^{-1} \frac{\partial \gamma}{\partial \tilde{\theta}}(q), \\
& a_{3}(q) \stackrel{\text { def }}{=} \gamma(q)^{-1} \frac{\partial \gamma}{\partial \tilde{r}}(q) .
\end{aligned}
$$

Lemma 2. With the hypotheses (H-0) to (H-3), there exist positive constants $\alpha, \lambda, K_{2}$, and $K_{3}$ such that, for $q, \tilde{q} \in Q$, the sesquilinear form $\tilde{\sigma}(q)(\cdot, \cdot)$ satisfies the following inequalities for all $\varphi, \psi \in \tilde{V}$ :

$$
\begin{aligned}
\tilde{\sigma}(q)(\psi, \psi) & \geq \alpha|\psi|_{\tilde{V}}^{2} \\
|\tilde{\sigma}(q)(\varphi, \psi)| & \leq K_{2}|\varphi|_{\tilde{V}}|\psi|_{\tilde{V}} \\
|\tilde{\sigma}(q)(\varphi, \psi)-\tilde{\sigma}(\tilde{q})(\varphi, \psi)| & \leq\left. K_{3} d(q, \tilde{q})\right|_{\tilde{V}}|\psi|_{\tilde{V}}
\end{aligned}
$$

where

$$
d(q, \tilde{q}) \rightarrow 0 \quad \text { as } \quad|q-\tilde{q}| \rightarrow 0 .
$$

Furthermore, $\alpha, K_{2}$, and $K_{3}$ can be chosen as constants which are independent of the parameter vector $q$.

Proof. From (21), the associated quadratic form is

$$
\begin{aligned}
& a_{1}(q) \xi_{1}^{2}+2 a_{2}(q) \xi_{1} \xi_{2}+a_{3}(q) \xi_{2}^{2} \\
& =\gamma^{-1}\left(\frac{\partial \gamma}{\partial \tilde{r}}\right)^{-1}\left[\left\{\gamma^{2}+\left(\frac{\partial \gamma}{\partial \tilde{\theta}}\right)^{2}\right\} \xi_{1}^{2}-2 \frac{\partial \gamma}{\partial \tilde{r}} \frac{\partial \gamma}{\partial \tilde{\theta}} \xi_{1} \xi_{2}+\left(\frac{\partial \gamma}{\partial \tilde{r}}\right)^{2} \xi_{2}^{2}\right] .
\end{aligned}
$$

For any quadratic form $Q F=a \xi_{1}^{2}-2 b \xi_{1} \xi_{2}+c \xi_{2}^{2}$ with $a>0, c>0$, completion of the square arguments yield $Q F \geq \xi_{1}^{2}\left(a c-b^{2}\right) / c$ and $Q F \geq \xi_{2}^{2}\left(a c-b^{2}\right) / a$ so that

$$
2 Q F \geq \frac{a c-b^{2}}{c} \xi_{1}^{2}+\frac{a c-b^{2}}{a} \xi_{2}^{2} .
$$


Choosing

$$
a=\gamma^{2}+\left(\frac{\partial \gamma}{\partial \tilde{\theta}}\right)^{2}, \quad b=\frac{\partial \gamma}{\partial \tilde{r}} \frac{\partial \gamma}{\partial \tilde{\theta}} \quad \text { and } \quad c=\left(\frac{\partial \gamma}{\partial \tilde{r}}\right)^{2}
$$

we easily find that

$$
\begin{aligned}
& a_{1}(q) \xi_{1}^{2}+2 a_{2}(q) \xi_{1} \xi_{2}+a_{3}(q) \xi_{2}^{2} \\
& \quad \geq \frac{1}{2} \gamma\left(\frac{\partial \gamma}{\partial \tilde{r}}\right)^{-1}\left[\xi_{1}^{2}+\left(\frac{\partial \gamma}{\partial \tilde{r}}\right)^{2}\left\{\gamma^{2}+\left(\frac{\partial \gamma}{\partial \tilde{\theta}}\right)^{2}\right\}^{-1} \xi_{2}^{2}\right]
\end{aligned}
$$

Note that

$$
\frac{\partial \gamma}{\partial \tilde{r}}=\frac{c(\theta, q)-l(\theta)}{\bar{c}-l(\theta)}
$$

and from (H-2) and (19), there exists a constant $C_{2}$ independent of $q$ such that

$$
\gamma{\frac{\partial \gamma^{-1}}{\partial \tilde{r}}}^{-1} \geq \frac{\beta_{1}\{\bar{c}-l(\theta)\}}{\beta_{1}-l(\theta)} \geq C_{2}
$$

where

$$
C_{2}=\min _{\theta \in[0,2 \pi]} \frac{\beta_{1}\{\bar{c}-l(\theta)\}}{\beta_{1}-l(\theta)} .
$$

With (H-1), there exists a constant $C_{3}$ such that

$$
\frac{\partial \gamma^{2}}{\partial \tilde{r}}\left\{\gamma^{2}+\left(\frac{\partial \gamma}{\partial \tilde{\theta}}\right)^{2}\right\}^{-1} \geq C_{3}
$$

where

$$
C_{3}=\min _{\theta \in[0,2 \pi]}\left\{\frac{\left.\beta_{2}-l(\theta)\right\}}{\bar{c}-l(\theta)}\right\}^{2}\left[\max _{\theta \in[0,2 \pi]}\{l(\theta)\}^{2}+\sup _{q \in Q} \sup _{\tilde{x} \in \tilde{G}}\left|\frac{\partial \gamma}{\partial \tilde{\theta}}\right|^{2}\right]^{-1}
$$

can be chosen independent of $q$ ( here we use (H-0) and (H-3) ). Thus if we choose

$$
\alpha=\frac{1}{2} C_{2} \min \left(1, C_{3}\right),
$$

we obtain the coercivity $(22)$ of the sesquilinear form $\tilde{\sigma}(q)(\cdot, \cdot)$. To prove the boundedness, we note that

$$
|\tilde{\sigma}(q)(\varphi, \psi)| \leq 4 \sup _{i \leq 3, \tilde{x} \in \tilde{G}}\left|a_{i}(q, \tilde{x})\right||\varphi|_{\tilde{V}}|\psi|_{\tilde{V}}
$$

From (H-0) - (H-3), we have

$$
\sup _{\tilde{x} \in \tilde{G}}\left|a_{i}(q, \tilde{x})\right|<K_{2} \quad \text { for } \quad i=1,2,3
$$


where $K_{2}$ is some constant independent of $q$. This implies the boundedness (23). To establish the continuity property, we note that, for any $q, \tilde{q} \in Q$,

$$
\begin{aligned}
& |\tilde{\sigma}(q)(\varphi, \psi)-\tilde{\sigma}(\tilde{q})(\varphi, \psi)| \\
& \leq \mid \int_{0}^{2 \pi} \int_{\bar{c}}^{l(\theta)}\left[\left\{a_{1}(q)-a_{1}(\tilde{q})\right\} \frac{\partial \varphi}{\partial \tilde{r}} \frac{\partial \psi}{\partial \tilde{r}}+\left\{a_{2}(q)-a_{2}(\tilde{q})\right\}\left(\frac{\partial \varphi}{\partial \tilde{r}} \frac{\partial \psi}{\partial \tilde{\theta}}+\frac{\partial \varphi}{\partial \tilde{\theta}} \frac{\partial \psi}{\partial \tilde{r}}\right)\right. \\
& \left.\quad+\left\{a_{3}(q)-a_{3}(\tilde{q})\right\} \frac{\partial \varphi}{\partial \tilde{\theta}} \frac{\partial \psi}{\partial \tilde{\theta}}\right] d \tilde{r} d \tilde{\theta} \mid .
\end{aligned}
$$

Under the hypotheses $(\mathbf{H}-\mathbf{1})$ and $(\mathbf{H}-3)$, we can argue that $a_{i}(q), i=1,2,3$, are continuous in $L_{\infty}(\tilde{G})$. We can thus infer the continuity of the sesquilinear form (18) with respect to $q \in Q$ and the proof of Lemma 2 is thus completed.

Lemma 3. Let

$$
\tilde{u}=u \circ T(q), \tilde{\phi}=\phi \circ T(q), \text { and } \tilde{\phi}^{*}=\phi^{*} \circ T(q)
$$

be the transformed system state, the transformed solution, and the transformed function associated with $\phi^{*}$, respectively. Then, for every $q \in Q$, there exists a unique solution

$$
\tilde{u}=\tilde{\phi}-\tilde{\phi}^{*} \in \tilde{V}
$$

in the sense that

$$
\tilde{\sigma}(q)(\tilde{u}, \psi)=-\tilde{\sigma}(q)\left(\tilde{\phi}^{*}, \psi\right) \quad \text { for } \psi \in \tilde{V}
$$

Moreover the solution $\tilde{u}$ in the system on $\tilde{G}$ is bounded in $\tilde{V}$ uniformly in $q \in Q$.

Proof. Using Lemma 2 and from (18), (21), and (20), we have that, for $\varphi \in V_{q}$ and $\tilde{\varphi}=\varphi \circ T(q)$,

$$
\begin{aligned}
|\varphi|_{V_{q}}^{2} & =\int_{0}^{2 \pi} \int_{c(\theta, q)}^{l(\theta)}\left\{r\left|\frac{\partial \varphi}{\partial r}\right|^{2}+\frac{1}{r}\left|\frac{\partial \varphi}{\partial \theta}\right|^{2}\right\} d r d \theta \\
& =\int_{0}^{2 \pi} \int_{\bar{c}}^{l(\theta)}\left\{a_{1}(q)\left|\frac{\partial \tilde{\varphi}}{\partial \tilde{r}}\right|^{2}+2 a_{2}(q) \frac{\partial \tilde{\varphi}}{\partial \tilde{r}} \frac{\partial \tilde{\varphi}}{\partial \tilde{\theta}}+a_{3}(q)\left|\frac{\partial \tilde{\varphi}}{\partial \tilde{\theta}}\right|^{2}\right\} d \tilde{r} d \tilde{\theta} \\
\geq \alpha|\tilde{\varphi}|_{\tilde{V}}^{2} . &
\end{aligned}
$$

Similarly, by virtue of Lemma 2 , it can be easily shown that

$$
|\varphi|_{V_{q}}^{2} \leq k_{2}|\tilde{\varphi}|_{\tilde{V}}^{2}
$$

where $k_{2}$ does not depend on $q \in Q$. This implies the $V_{q}$-norm is equivalent to the norm in $\tilde{V}$ uniformly in $q \in Q$. Thus we have the solution $\tilde{u}(q)=u(q) \circ T(q)$ in the sense of (26) satisfying

$$
|\tilde{u}(q)|_{\tilde{V}} \leq \alpha^{-\frac{1}{2}}|u(q)|_{V_{q}} \leq \alpha^{-\frac{1}{2}} K_{1}\left|\phi^{*}\right|_{V_{q}} \leq \alpha^{-\frac{1}{2}} K_{1} k_{2}^{-\frac{1}{2}}\left|\tilde{\phi}^{*}\right|_{\tilde{V}}
$$


which follows from Lemma 1 and verifies the claim of Lemma 3. $\tilde{V}$.

Lemma 4. With the hypotheses $(\mathbf{H}-\mathbf{0})$ to $(\mathbf{H}-\mathbf{3}), q \rightarrow \tilde{u}(q)$ is continuous from $Q$ to

Proof. Let $q^{k} \rightarrow q$ in $Q$ and let $\tilde{u}\left(q^{k}\right), \tilde{u}(q)$ be corresponding solution of (26). That is,

$$
\begin{array}{rll}
\tilde{\sigma}\left(q^{k}\right)\left(\tilde{u}\left(q^{k}\right), \psi\right) & =-\tilde{\sigma}(q)\left(\tilde{\phi}^{*}, \psi\right) & \text { for } \psi \in \tilde{V} \\
\tilde{\sigma}(q)(\tilde{u}(q), \psi) & =-\tilde{\sigma}(q)\left(\tilde{\phi}^{*}, \psi\right) & \text { for } \psi \in \tilde{V} .
\end{array}
$$

Substracting (28) from (27), we have

$$
\tilde{\sigma}\left(q^{k}\right)\left(\tilde{u}\left(q^{k}\right), \psi\right)-\tilde{\sigma}(q)(\tilde{u}(q), \psi)=\left[\tilde{\sigma}(q)-\tilde{\sigma}\left(q^{k}\right)\right]\left(\tilde{\phi}^{*}, \psi\right) \quad \text { for all } \psi \in \tilde{V} \text {. }
$$

Then we may write

$$
\tilde{\sigma}\left(q^{k}\right)\left(\tilde{u}\left(q^{k}\right)-\tilde{u}(q), \psi\right)+\left[\tilde{\sigma}\left(q^{k}\right)-\tilde{\sigma}(q)\right](\tilde{u}(q), \psi)=\left[\tilde{\sigma}(q)-\tilde{\sigma}\left(q^{k}\right)\right]\left(\tilde{\phi}^{*}, \psi\right) \quad \text { for } \psi \in \tilde{V} .
$$

We note that while $\tilde{\phi}^{*} \notin \tilde{V}$ - because of its boundary values it is only in $H^{1}(\tilde{G})$ it is readily seen from the proof of Lemma 2 that (24) holds for $\varphi=\phi^{*}$. Choosing as $\psi \equiv \tilde{u}\left(q^{k}\right)-\tilde{u}(q)$ in $\tilde{V}$ and using (22) and (24) in Lemma 2, we find from (29)

$$
\alpha\left|\tilde{u}\left(q^{k}\right)-\tilde{u}(q)\right|_{\tilde{V}}^{2} \leq d\left(q^{k}, q\right)\left\{\left|\tilde{\phi}^{*}\right|_{\tilde{V}}+|\tilde{u}(q)|_{\tilde{V}}\right\}\left|\tilde{u}\left(q^{k}\right)-\tilde{u}(q)\right|_{\tilde{V}},
$$

or

$$
\alpha\left|\tilde{u}\left(q^{k}\right)-\tilde{u}(q)\right|_{\tilde{V}} \leq d\left(q^{k}, q\right)\left\{\left|\tilde{\phi}^{*}\right|_{\tilde{V}}+|\tilde{u}(q)|_{\tilde{V}}\right\} \quad \longrightarrow 0 \quad \text { as } q^{k} \rightarrow q .
$$

This yields the desired continuity.

From (9), the system output has the form

$$
\begin{aligned}
&\left\{Y_{p}^{i}\right\}=-\frac{\sigma_{0} \mu_{0} \Delta}{4 \pi\left|S_{p}^{i}\right|} \int_{S_{p}^{i}} \int_{0}^{2 \pi} \int_{c(\theta, q)}^{l(\theta)} r\left\{\sin \left(\theta-\theta^{\prime}\right) \frac{\partial \phi}{\partial r^{\prime}}-\frac{1}{r^{\prime}} \cos \left(\theta+\theta^{\prime}\right) \frac{\partial \phi}{\partial \theta^{\prime}}\right\} \\
& \times\left\{r^{2}+r^{\prime^{2}}-2 r r^{\prime} \cos \left(\theta+\theta^{\prime}\right)+h^{2}\right\}^{-\frac{3}{2}} d r d \theta d r^{\prime} d \theta^{\prime} \\
& \text { for } i=1,2, \cdots m .
\end{aligned}
$$

From (30), the observation output for the transformed system can be written as

$$
\tilde{Y}(q)=\mathcal{H}(q) \tilde{\phi}(q)
$$

where $\mathcal{H}(q): \tilde{V} \rightarrow R^{m}$ is given by

$$
[\mathcal{H}(q) \tilde{\varphi}]_{i}=-\frac{\sigma_{0} \mu_{0} \Delta}{4 \pi\left|S_{p}^{i}\right|} \int_{S_{p}^{i}} \int_{0}^{2 \pi} \int_{\bar{c}}^{l(\theta)}\left\{h_{1}\left(r, \theta, \tilde{r}^{\prime}, \tilde{\theta}^{\prime} ; q\right) \frac{\partial \tilde{\varphi}}{\partial \tilde{r}^{\prime}}-h_{2}\left(r, \theta, \tilde{r}^{\prime}, \tilde{\theta}^{\prime} ; q\right) \frac{\partial \tilde{\varphi}}{\partial \tilde{\theta}^{\prime}}\right\} d r d \theta d \tilde{r}^{\prime} d \tilde{\theta}^{\prime}
$$




$$
\text { for } \quad i=1,2, \cdots, m
$$

where

$$
\begin{aligned}
h_{1}\left(r, \theta, \tilde{r}^{\prime}, \tilde{\theta}^{\prime} ; q\right)= & \bar{h}\left(r, \theta, \tilde{r}^{\prime}, \tilde{\theta}^{\prime} ; q\right)\left[\sin \left(\theta-\tilde{\theta}^{\prime}\right)\right. \\
& \left.+\left\{\gamma\left(\tilde{r}^{\prime}, \tilde{\theta} ; q\right)\right\}^{-1} \frac{\partial \gamma\left(\tilde{r}^{\prime}, \tilde{\theta}^{\prime} ; q\right)}{\partial \tilde{\theta}^{\prime}} \cos \left(\theta+\tilde{\theta}^{\prime}\right)\right], \\
h_{2}\left(r, \theta, \tilde{r}^{\prime}, \tilde{\theta}^{\prime} ; q\right)= & \bar{h}\left(r, \theta, \tilde{r}^{\prime}, \tilde{\theta}^{\prime} ; q\right)\left\{\gamma\left(\tilde{r}^{\prime}, \tilde{\theta} ; q\right)\right\}^{-1} \frac{\partial \gamma\left(\tilde{r}^{\prime}, \tilde{\theta}^{\prime} ; q\right)}{\partial \tilde{r}^{\prime}} \cos \left(\theta+\tilde{\theta}^{\prime}\right), \\
\bar{h}\left(r, \theta, \tilde{r}^{\prime}, \tilde{\theta}^{\prime} ; q\right)= & r\left[r^{2}+\left\{\gamma\left(\tilde{r}^{\prime}, \tilde{\theta} ; q\right)\right\}^{2}-2 r \gamma\left(\tilde{r}^{\prime}, \tilde{\theta} ; q\right) \cos \left(\theta+\tilde{\theta}^{\prime}\right)+h^{2}\right]^{-\frac{3}{2}}
\end{aligned}
$$

Lemma 4. With the hypotheses $(\mathbf{H}-\mathbf{0})$ to $(\mathbf{H - 3})$, the mapping $q \rightarrow Y(q)$ is continuous from $Q$ to $R^{m}$.

Proof. Taking into account that the operator $\mathcal{H}(q)$ belongs to $\mathcal{L}\left(\tilde{V} ; R^{m}\right)$ for each $q \in Q$, we see that the above statement follows directly from Lemma 3 .

Thus the identification problem of interest is defined by the fit-to-data functional

$$
E(q)=\frac{1}{2}\left|\mathcal{H}(q) \tilde{\phi}(q)-Y_{d}\right|^{2}
$$

where $\left\{Y_{d}\right\}$ denotes the given observed data. The identification problem can then be stated as follows:

(IDP): Find $q^{*} \in Q$ which minimizes $E(q)$ given by (32) subject to (25), (26), and $(31)$.

Theorem 1. Under the hypotheses (H-0)- (H-4), the problem (IDP) has at least one solution $q^{*} \in Q$. $Q$.

Proof. With Lemmas 3 to 5, the above statements follows from the compactness of

\section{APPROXIMATE IDENTIFICATION PROBLEMS}

The computational scheme we propose below is based on the use of a finite element Galerkin approach to construct a sequence of finite dimensional approximating identification problems. To approximate problem (IDP), we choose a sequence of finite dimensional subspaces $H^{N} \subset \tilde{V}$ such that

$$
\left|P^{N} \psi-\psi\right|_{\tilde{V}} \longrightarrow 0 \quad \text { as } \quad N \rightarrow \infty \quad \text { for } \quad \psi \in \tilde{V}
$$


where $P^{N}$ is the orthogonal projection of $\tilde{H}=L_{2}(\tilde{G})$ onto $H^{N}$. The approximating system is defined for $\tilde{u}^{N}(q) \in H^{N}$ by

$$
\tilde{\sigma}(q)\left(\tilde{u}^{N}(q), \psi^{N}\right)=-\tilde{\sigma}(q)\left(\tilde{\phi}^{*}, \psi^{N}\right) \quad \text { for } \psi^{N} \in H^{N} .
$$

From (31), the observation output for the approximate system (34) can be represented as

$$
\tilde{Y}^{N}(q)=\mathcal{H}^{N}(q) \tilde{\phi}^{N}(q)
$$

where $\mathcal{H}^{N}(q): H^{N} \rightarrow R^{m}$ and where $\tilde{\phi}^{N}(q)=\tilde{u}^{N}+\tilde{\phi}^{*}$. Then we seek to solve the approximate identification problem:

$(\mathbf{A I D P})^{N}:$ Find $\hat{q}^{N} \in Q$ which minimizes

$$
E^{N}(q)=\frac{1}{2}\left|\mathcal{H}^{N}(q) \tilde{\phi}^{N}(q)-Y_{d}\right|^{2}
$$

subject to the finite dimensional system (34), (35).

Lemma 5. Let $q^{N} \rightarrow q \in Q$. Then $\tilde{u}^{N}\left(q^{N}\right) \longrightarrow \tilde{u}(q) \in \tilde{V}$.

Proof. We have

$$
\begin{aligned}
\tilde{\sigma}\left(q^{N}\right)\left(\tilde{u}^{N}\left(q^{N}\right), \psi^{N}\right) & =-\tilde{\sigma}\left(q^{N}\right)\left(\tilde{\phi}^{*}, \psi^{N}\right) \quad \text { for } \psi^{N} \in H^{N} \\
\tilde{\sigma}(q)(\tilde{u}(q), \psi) & =-\tilde{\sigma}(q)\left(\tilde{\phi}^{*}, \psi\right) \quad \text { for } \psi \in \tilde{V}
\end{aligned}
$$

Then it follows that

$$
\left|\tilde{u}^{N}\left(q^{N}\right)-\tilde{u}(q)\right|_{\tilde{V}} \leq\left|\tilde{u}^{N}\left(q^{N}\right)-P^{N} \tilde{u}(q)\right|_{\tilde{V}}+\left|P^{N} \tilde{u}(q)-\tilde{u}(q)\right|_{\tilde{V}}
$$

Hence from (33) it suffices to prove

$$
\left|\tilde{u}^{N}\left(q^{N}\right)-P^{N} \tilde{u}(q)\right|_{\tilde{V}} \longrightarrow 0 \quad \text { for } \quad q^{N} \rightarrow q \in Q \text { as } N \rightarrow \infty .
$$

Taking $\psi=\psi^{N}$ in (37) and subtracting this from (36), we obtain

$$
\tilde{\sigma}\left(q^{N}\right)\left(\tilde{u}^{N}\left(q^{N}\right), \psi^{N}\right)-\tilde{\sigma}(q)\left(\tilde{u}(q), \psi^{N}\right)=\left[\tilde{\sigma}(q)-\tilde{\sigma}\left(q^{N}\right)\right]\left(\tilde{\phi}^{*}, \psi^{N}\right) \quad \text { for } \psi^{N} \in H^{N} \text {. }
$$

Furthermore we have

$$
\begin{gathered}
\tilde{\sigma}\left(q^{N}\right)\left(\tilde{u}^{N}\left(q^{N}\right)-P^{N} \tilde{u}(q), \psi^{N}\right)+\tilde{\sigma}\left(q^{N}\right)\left(P^{N} \tilde{u}(q)-\tilde{u}(q), \psi^{N}\right) \\
+\left[\tilde{\sigma}\left(q^{N}\right)-\tilde{\sigma}(q)\right]\left(\tilde{u}(q), \psi^{N}\right)=\left[\tilde{\sigma}(q)-\tilde{\sigma}\left(q^{N}\right)\right]\left(\tilde{\phi}^{*}, \psi^{N}\right) .
\end{gathered}
$$

Choosing $\psi^{N}=\Delta^{N}=\tilde{u}^{N}\left(q^{N}\right)-P^{N} \tilde{u}(q)$, we find that

$$
\begin{aligned}
& \tilde{\sigma}\left(q^{N}\right)\left(\Delta^{N}, \Delta^{N}\right)+\tilde{\sigma}\left(q^{N}\right)\left(P^{N} \tilde{u}(q)-\tilde{u}(q), \Delta^{N}\right) \\
& +\left[\tilde{\sigma}\left(q^{N}\right)-\tilde{\sigma}(q)\right]\left(\tilde{u}(q), \Delta^{N}\right)=\left[\tilde{\sigma}(q)-\tilde{\sigma}\left(q^{N}\right)\right]\left(\tilde{\phi}^{*}, \Delta^{N}\right) .
\end{aligned}
$$


From this we obtain

$$
\alpha\left|\Delta^{N}\right|_{\tilde{V}}^{2} \leq K_{2}\left|P^{N} \tilde{u}(q)-\tilde{u}(q)\right|_{\tilde{V}}\left|\Delta^{N}\right|_{\tilde{V}}+d\left(q^{N}, q\right)|\tilde{u}(q)|_{\tilde{V}}\left|\Delta^{N}\right|_{\tilde{V}}+d\left(q, q^{N}\right)\left|\tilde{\phi}^{*}\right|_{\tilde{V}}\left|\Delta^{N}\right|_{\tilde{V}} .
$$

Consequently, we have

$$
\alpha\left|\Delta^{N}\right|_{\tilde{V}} \leq K_{2}\left|P^{N} \tilde{u}(q)-\tilde{u}(q)\right|_{\tilde{V}}+d\left(q^{N}, q\right)\left(|\tilde{u}(q)|_{\tilde{V}}+\left|\tilde{\phi}^{*}\right|_{\tilde{V}}\right) .
$$

Thus, given any $q^{N} \rightarrow q \in Q$, it follows from (33) that $\Delta^{N} \rightarrow 0$ as $N \rightarrow \infty$.

Since it can readily be shown that the approximate solution $\tilde{\phi}^{N}$ depends continuously on $q$, solutions exist to the problem $(\text { AIDP })^{N}$ for each $N$. Our convergence results for the approximating identification problem $(\text { AIDP })^{N}$ are summarized in the following theorem which follows from standard arguments ( e.g., see [7] ).

Theorem 2. Suppose that hypotheses $(\mathbf{H}-\mathbf{0})$ to $(\mathbf{H}-\mathbf{3})$ hold and let $\hat{q}^{N}$ be a solution of the problem (AIDP) $)^{N}$. Then the sequence $\left\{\hat{q}^{N}\right\}$ admits a convergent subsequence $\left\{\hat{q}^{N_{k}}\right\}$ with $\hat{q}^{N_{k}} \rightarrow q^{*}$ as $N_{k} \rightarrow \infty$. Moreover, $q^{*}$ is a solution of the problem (IDP).

\section{COMPUTATIONAL METHOD}

It is rather straightforward to develop computational methods based on the preceding foundations. For example, we may consider a linear spline approximation of a parametrized function for $c(q, \theta)$. Let $S^{M}$ be the set of piecewise linear splines ( see [5] for more details ) with the knot sequence $S_{\Delta}^{M}=\{i 2 \pi / M\}_{i=0}^{M}$ and basis elements $\left\{B_{i}^{M}\right\}_{i=0}^{M}$. Then we approximate the unknown defect shape function by

$$
c(q, \theta)=\sum_{i=0}^{M} q_{i}^{M} B_{i}^{M}(\theta) \quad \text { for } \theta \in[0,2 \pi] .
$$

In order to ensure (H-0), we impose constraints on $Q$ expressed by

$$
Q=\left\{q \in R^{M} \mid \beta_{1} \leq q_{i} \leq \beta_{2} i=1,2, \cdots, M\right\} .
$$

It is clear that the defect shape function defined by (38) satisfies the hypotheses (H-2) and (H-3). Let us choose $\cup_{N=1}^{\infty}\left\{\psi_{i}^{N}\right\}_{i=1}^{N}$ as a set of basis functions in $\tilde{V}$. That is, for each $N,\left\{\psi_{i}^{N}\right\}_{i=1}^{N}$ are linearly independent and $\cup_{N} \operatorname{span}\left\{\psi_{i}^{N}\right\}_{i=1}^{N}$ is dense in $H_{1}(\tilde{G})$. Then the approximation subspaces can be chosen as $H^{N}=\operatorname{span}\left\{\psi_{i}^{N}\right\}_{i=1}^{N}$. An approximate solution can be defined by

$$
\tilde{\phi}^{N}=\tilde{u}^{N}+\tilde{\phi}^{*}=\sum_{i=1}^{N} w_{i}^{N} \psi_{i}^{N}+\tilde{\phi}^{*}
$$

where the coefficient vector $w^{N}=\left\{w_{i}^{N}\right\}$ is chosen such that, for $j=1,2, \cdots, N$,

$$
\tilde{\sigma}(q)\left(\psi_{j}^{N}, \sum_{i=1}^{N} w_{i}^{N} \psi_{i}^{N}\right)=-\tilde{\sigma}(q)\left(\tilde{\phi}^{*}, \psi_{j}^{N}\right) .
$$


Hence the system can be approximated by solving the linear system

$$
L^{N}(q) w^{N}=f^{N}(q)
$$

where

$$
\begin{aligned}
& {\left[L^{N}(q)\right]_{i, j} \stackrel{\text { def }}{=} \tilde{\sigma}(q)\left(\psi_{j}^{N}, \psi_{i}^{N}\right) \quad \text { for } \quad i, j=1,2, \cdots, N \text {, }} \\
& {\left[f^{N}(q)\right]_{i} \stackrel{\text { def }}{=}-\tilde{\sigma}(q)\left(\tilde{\phi}^{*}, \psi_{i}^{N}\right) \quad \text { for } \quad i=1,2, \cdots, N .}
\end{aligned}
$$

The corresponding output can be computed as

$$
Y^{N}(q)=\mathcal{H}^{N}(q) w^{N}(q)+g(q)
$$

where

$$
\left[\mathcal{H}^{N}(q)\right]_{i, j} \stackrel{\text { def }}{=}[\mathcal{H}(q)]_{i} \psi_{j}^{N} \quad \text { for } \quad i=1,2, \cdots, m ; j=1,2, \cdots, N,
$$

and where

$$
[g(q)]_{i} \stackrel{\text { def }}{=}[\mathcal{H}(q)]_{i} \tilde{\phi}^{*} \quad \text { for } \quad i=1,2, \cdots, m,
$$

respectively. Associated with the problem $(\text { AIDP) })^{N}$, the problem is to find the parameter $\hat{q}^{N} \in Q$ which minimizes

$$
E^{N}(q)=\frac{1}{2}\left|Y^{N}(q)-Y_{d}\right|^{2}
$$

subject to the finite dimensional system (39), (40), and (41). Solutions to the problem (AIDP) $)^{N}$ exist for each $N$. In order to implement the identification scheme, it is necessary to evaluate the gradient of the cost functional (42). Let $\hat{q}^{N}$ be an optimal solution of the problem for (42). Then a necessary condition for $\hat{q}^{N}$ to be optimal is characterized by

$$
\nabla_{q} E^{N}\left(\hat{q}^{N}\right) \cdot\left(q-\hat{q}^{N}\right) \geq 0 \quad \text { for all } q \in Q,
$$

where $\nabla_{q}$ denotes the gradient of $E^{N}(q)$ with respect to $q$. From (42), the gradient of $E^{N}(q)$ becomes, for $i=1,2, \cdots, M$,

$$
\left[\nabla_{q} E^{N}\left(\hat{q}^{N}\right)\right]_{i}=\left\langle-\frac{\partial K^{N}(q)}{\partial q_{i}} w^{N}+\frac{\partial f^{N}}{\partial q_{i}}, v^{N}\right\rangle+\left\langle-\frac{\partial \mathcal{H}^{N}(q)}{\partial q_{i}} w^{N}+\frac{\partial g}{\partial q_{i}}, Y^{N}(q)-Y_{d}\right\rangle
$$

where $v^{N}$ is the solution of the adjoint system,

$$
\left[K^{N}(q)\right]^{T} v^{N}=\left[\mathcal{H}^{N}(q)\right]^{T}\left(Y^{N}(q)-Y_{d}\right) .
$$

With (43), numerical optimization techniques are readily applicable to the problem for (42) ( see [6] and the references therein ).

\section{CONCLUDING REMARKS}


The results given here, while applied to a particular domain described by the interior boundary $c(\theta, q)$ and exterior boundary $l(\theta)$, are rather generic in nature for elliptic problems with unknown domains. The techniques used here ( and the necessary assumptions on the problems ) represent a rather nontrivial improvement of the results and ideas presented in Chapter VI of Banks/Kunisch [7] for parameter estimation convergence in elliptic problems. In particular, the presentation for the elliptic system in [7] requires a nonvanishing positive coefficients in the lowest order term (i.e., $c(x) \geq \bar{c}>0$, see (1.3) of [7]) in $L(q) u=\nabla \cdot(a \nabla u)+b \cdot \nabla u+c u$ that can be replaced with the techniques used here ( The results of [7] cannot be applied directly to the problem we treated here). While the ideas are in the sprit of [7], the detailed arguments are more similar to an elliptic version of the hyperbolic system presentation of Chapter 5 of [8] ( compare, in particular, (A3N) and (H7) of pages 124 in [8] with our conditions (33) and (24).

The generic nature of the results presented here is primarily manifested in the nature of the transformation $T(q): \tilde{G} \rightarrow G_{q}$ between the reference domain $\tilde{G}$ and the unknown domain $G_{q}$. As long as this map satisfies requirements on $|\nabla T(q)|$ and $[\nabla T(q)]^{-1}$ needed above and yields a transformed system with appropriately smooth coefficients $a_{i}(q)(i=1,2,3)$, the arguments remain valid even if the unknown domain is not "annular" in nature with unknown boundary. For example, as shown in Fig. 5, a parallelpiped ( slit or crack line ) shaped damage and structure should be amenable to the arguments given here (in this case we would use rectangular coordinates in the place of polar coordinates ) where now $q$ parametrizes the slit or crack-like damage ( similar to the considerations in [3][4] on thermal nondestructive evaluation ).

\section{Acknowledgements}

The authors would like to express their sincere appreciation to Dr. Naoko Kasai ( Electrotechnical Laboratory, Tsukuba, JAPAN ) for numerous conversations and encouragement during the course of this research. This research was supported in part (H.T.Banks) by the U.S. Air Force Office of Scientific Research under grant AFOSR F49620-98-1-0180 and was supported in part (F. Kojima) by the Research Committee on Nondestructive Evaluation for Assessment of Material Degradation in Nuclear Reactor Pressure Vessels of the Japan Society of Applied Electromagnetics and Mechanics. Parts of this research

were carried out while the first author was a visiting scientist at the Centre Émile Borel, Institut Henri Poincaré, Paris.

\section{References}

1. N. Kasai, S. Nakayama and H. Yamakawa, A possibility for NDE of magnetic material using SQUID, Studies in Applied Electromagnetics and Mechanics, (1997) 12, $95-101$. 
2. P.P. Silvester and R.L. Ferrari, Finite Elements for Electrical Engineers, Cambridge University Press, Cambridge, 1983.

3. H.T. Banks and F. Kojima, Boundary shape identification problems in two-dimensional domains related to thermal testing of materials, Quart. Appl. Math., (1989) 47, 273-293.

4. H.T. Banks, F. Kojima and W.P. Winfree, Boundary estimation problems arising in thermal tomography, Inverse Problems, (1990) 6, 897-921.

5. C. de Boor, A Practical Guide to Splines, Applied Mathematical Science, Vol.27, Springer, New York, 1978.

6. F. Kojima, Computational method for inverse problems in engineering sciences, Int. J. Applied Electromagnetics and Mechanics, (1997) 8 45-60.

7. H.T. Banks and K. Kunisch, Estimation Techniques for Distributed Parameter Systems, Birkhäuser, Boston, 1989.

8. H.T. Banks, R.C. Smith and Y. Wang, Smart Material Structures, Modeling, Estimation and Control, Masson, Paris, 1996.

9. O. Pironneau, Optimal Shape Design for Elliptic Systems, Springer-Verlag, New York, 1983. 

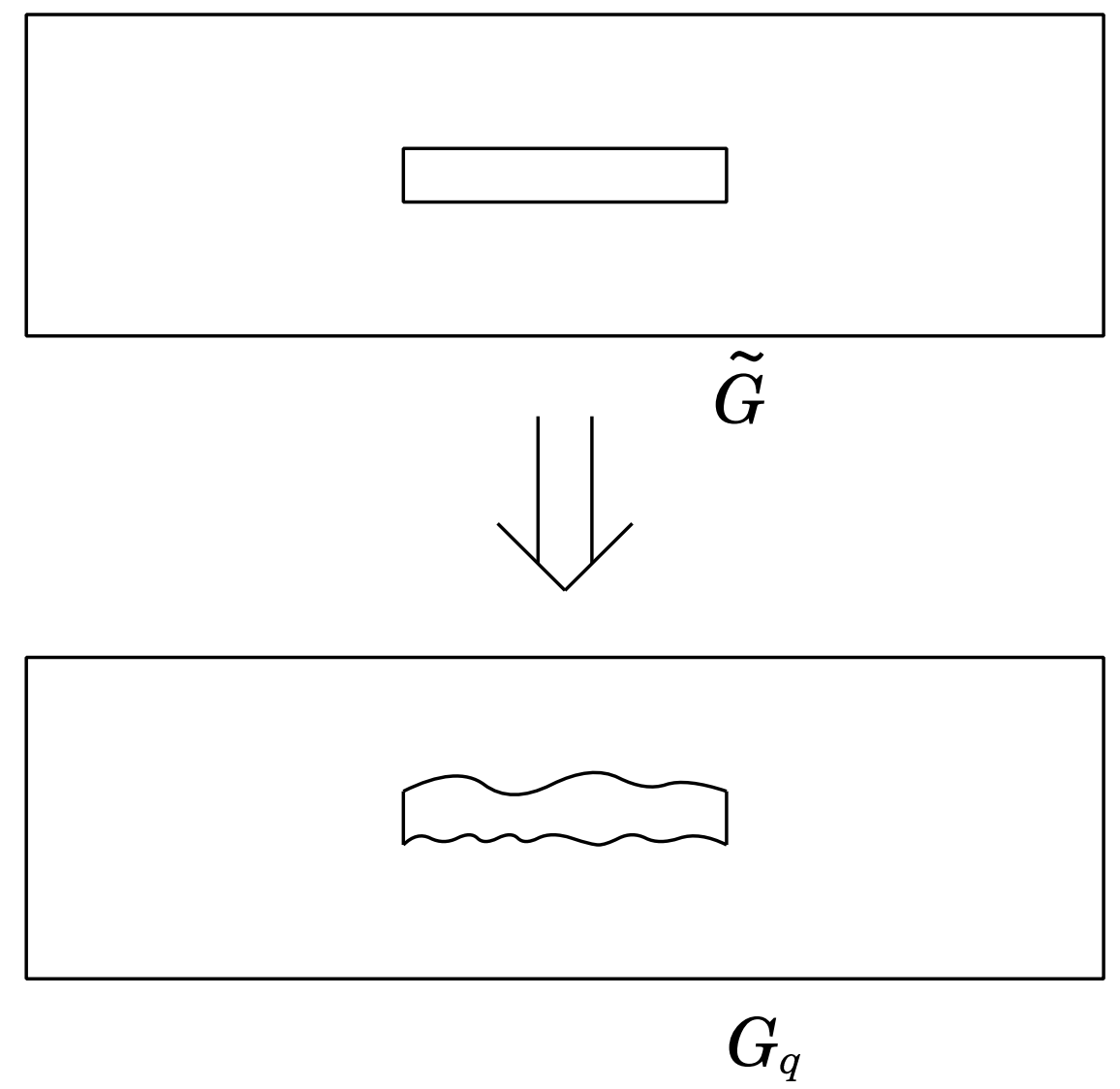

Fig. 5 Material with a defect of slit type and its reference domain shape 